\title{
N.m.r. study of the ferroelectric phase transition in a 70/30 mol\% copolymer of vinylidene fluoride (VF2) and trifluoroethylene (TrFE)
}

\author{
Authors: J.F. Legrand, P.J. Schuele, V. Hugo Schmidt
}

NOTICE: this is the author's version of a work that was accepted for publication in Polymer. Changes resulting from the publishing process, such as peer review, editing, corrections, structural formatting, and other quality control mechanisms may not be reflected in this document. Changes may have been made to this work since it was submitted for publication. A definitive version was subsequently published in Polymer, VOL\# 26, ISSUE\# 11, October 1985. DOI\# 10.1016/0032-3861(85)90286-1

J.F. Legrand, P.J. Schuele, V.H. Schmidt, and M. MInier, "N.m.r study of the ferroelectric phase transition in a 70/30 mole\% copolymer of vinylidene fluoride and trifluoroethylene," Polymer 26, 1683-1688 (1985)

http://dx.doi.org/10.1016/0032-3861(85)90286-1

Made available through Montana State University's ScholarWorks scholarworks.montana.edu 


\title{
N.m.r. study of the ferroelectric phase transition in a 70/30 mol\% copolymer of vinylidene fluoride $\left(\mathrm{VF}_{2}\right)$ and trifluoroethylene (TrFE)
}

\author{
J. F. Legrand $\ddagger$ P. J. Schuele and V. H. Schmidt \\ Montana State University, Bozeman, MT 59717, USA \\ and $M$. Minier* \\ Laboratoire de Spectrometrie Physique, Université de Grenoble 1, B.P. 87, 38402 Saint \\ Martin d'Heres Cedex, France
}

(Received 2 October 1984; revised 5 February 1985)

\begin{abstract}
Nuclear magnetic resonance (n.m.r.) studies of ${ }^{19} \mathrm{~F}$ nuclei in a $70 / 30 \mathrm{~mol} \%$ random copolymer of vinylidene fluoride and trifluoroethylene were performed at $9.14 \mathrm{MHz}$ and $20.0 \mathrm{MHz}$. The free induction decays (FIDs) were analysed in terms of two $T_{2}$ components attributed to the amorphous and crystalline portions of the polymer. The changes in crystallinity as well as the effects of the ferroelectric transition were observed during cycles of heating and cooling between $20^{\circ} \mathrm{C}$ and $140^{\circ} \mathrm{C}$. The crystalline component of the $F I D$ lengthens by a factor of 2 at $100^{\circ} \mathrm{C}$ on heating and decreases by this factor at $60^{\circ} \mathrm{C}$ on cooling, thus exhibiting the thermal hysteresis of this ferroelectric transition. The spin-lattice relaxation was also investigated. From measurements at $9.14 \mathrm{MHz}$ the observed longitudinal relaxation time $T_{1}$ appears to be dominated by the dynamics of the amorphous phase and exhibits no anomaly through the phase transition. However, from measurements at $20 \mathrm{MHz}$, well defined minima of $T_{1}$ were observed, which are associated with the ferroelectric transition (especially after repeated annealing of the samples). Results are discussed in terms of the crystalline phase structure, which appears dynamically disordered above the ferroelectric phase transition. An analogy is considered with the plastic phase transitions encountered in molecular crystals.
\end{abstract}

(Keywords: vinylidene fluoride-trifluoroethylene copolymers; ferroelectric phase transition; nuclear magnetic resonance; motional narrowing; plastic phase transition; crystallinity of polymers)

\section{INTRODUCTION}

Random copolymers of vinylidene fluoride and trifluoroethylene, with $\mathrm{VF}_{2}$ content ranging from 52 to $78 \mathrm{~mol} \%$ were extensively studied by Lovinger et al. ${ }^{1-3}$. These copolymers have ferroelectric transition temperatures, $T_{c}$, which increase with increasing $\mathrm{VF}_{2}$ content, from $70^{\circ} \mathrm{C}$ to $140^{\circ} \mathrm{C}$ for this composition range. Also, it is suggested that the absence of a distinct ferroelectric transition in the homopolymer $\mathrm{PVF}_{2}$ is due to prior melting at $185^{\circ} \mathrm{C}^{2}$.

In the $\mathrm{VF}_{2}-\mathrm{TrFE}$ copolymers, the transition is broad and exhibits considerable thermal hysteresis. Indeed, from X-ray data ${ }^{3}$, it appears to be a first-order phase transition (with coexistence of two distinct crystalline phases), but due to the very small size of the lamellar crystals embedded in the amorphous matrix ${ }^{4}$, the nucleation and growth of the new phase may occur over a finite temperature range. N.m.r. studies of the homopolymer $\mathrm{PVF}_{2}$ by McBrierty et al. were able to identify several types of molecular motions in the amorphous and $\alpha$ crystalline phases ${ }^{5}$ and also to analyse the orientation of the crystalline $\beta$ phase achieved by drawing and poling ${ }^{6}$. In a recent paper, they reported similar studies on a $52 / 48$ $\mathrm{mol}_{\%} \mathrm{VF}_{2}-\mathrm{TrFe}$ copolymer ${ }^{17}$.

Here we report some n.m.r. measurements on a 70 $30 \mathrm{~mol}^{\circ} \mathrm{VF}_{2}-\mathrm{TrFE}$ copolymer, in an effort to study the molecular motions both below and above $T_{c}$. At room

\footnotetext{
Deceased.

Being subjected to an intense d.c. electric polarizing field.

On leave from Université de Grenoble 1, France.
}

temperature, the crystalline phase of this copolymer is closely analogous to the ferroelectric $\beta$ phase of $\mathrm{PVF}_{2}$, with the molecular chains in a polar all-trans conformation (point group $m 2 m$ ). Upon heating, the ferroelectric phase transition occurs around $T_{\mathrm{c}} \simeq 115^{\circ} \mathrm{C}^{2}$, and in the paraelectric high temperature phase the molecular chains assume a partly disordered conformation consisting of irregular $T G T \bar{G}$ and $T T$ sequences, and are packed on an expanded hexagonal lattice ${ }^{3}$.

The main purpose of this ongoing work is to answer the following questions:

(1) Is the order-disorder phase transition associated with a definite molecular motion in the high temperature phase which freezes out in the low temperature phase?

(2) Is the disordered phase of higher symmetry due to a flipping motion of the chain segments between 6 or 3 symmetrical equilibrium positions which become inequivalent in the ordered phase?

(3) From 'symmetry-breaking' analysis several point groups are possible for the parent phase. The most likely are $6 / \mathrm{mmm}$ (centrosymmetric) and $\overline{6} 2 \mathrm{~m}$ (noncentrosymmetric). Which point group actually occurs?

The answers to these questions may help in understanding the properties of the ferroelectric phase and especially the poling mechanism of the material. Some of them can be elucidated using n.m.r. techniques.

\section{EXPERIMENTAL}

The random copolymer of vinylidene fluoride and 
trifluoroethylene was synthesized by Atochem Co. (France) and oriented samples were prepared at Thomson-CSF Laboratories by F. Micheron's group according to the following technique:

(i) quenching from the melt to $20^{\circ} \mathrm{C}$, producing unoriented crystalline $\beta$ phase of low crystallinity;

(ii) subsequent rolling (without poling) at $70^{\circ} \mathrm{C}$ with a draw ratio of $300 \%$, which provided semicrystalline copolymer films of $0.51 \mathrm{~mm}$ thickness, with biaxial orientation of the crystalline axes. It has been shown for the homopolymer $\mathrm{PVF}_{2}{ }^{7}$ that the rolling process highly orients the crystallite $c$ axes along the draw direction (labelled 1 in Figure 1 ), while, without simultaneous poling, it induces a preferential orientation of the polar $\underline{b}$ axes along the direction 2 (Figure l) which lies in the plane of the film.

For these preliminary experiments, unpoled specimens were preferred because macroscopic polarization of the films appears to induce additional changes in their texture: increase of the crystallinity ${ }^{3,17}$, decrease of the biaxial orientation ${ }^{6,7}$ and higher temperature of nucleation of the paraelectric phase $e^{1,17}$.

Twenty discs ( $9.5 \mathrm{~mm}$ in diameter) were cut and stacked in the nuclear magnetic resonance (n.m.r.) coil with their normals (direction 3) along the axis of the coil $\left(\mathrm{H}_{1}\right)$ and with the (direction 1) and (direction 2) axes $45^{\circ}$ to the static field $H_{0}$ (see Figure 1 ). This configuration gives a larger value for the transverse relaxation time $T_{2}$ from the crystalline phase as has also been reported for unpoled $\mathrm{PVF}_{2}$ by Douglass et al. ${ }^{6}$ and by Ishii et al. ${ }^{16}$. This occurs because such an orientation is close to the 'magic angle' which minimizes intramolecular line broadening from the $\mathrm{CF}_{2}$ and $\mathrm{CHF}$ groups.

In the first series of experiments, at M.S.U., we used a Matec pulse n.m.r. spectrometer operating at $9.14 \mathrm{MHz}$ to study the ${ }^{19} \mathrm{~F}$ resonance as a function of temperature from $24^{\circ} \mathrm{C}$ to $145^{\circ} \mathrm{C}$. The ${ }^{19} \mathrm{~F}$ resonance was chosen rather than the proton resonance because the abundance ratio of ${ }^{19} \mathrm{~F}$ to ${ }^{1} \mathrm{H}$ nuclei is 1.4 for this $70 / 30$ copolymer. In addition, the ${ }^{19} \mathrm{~F}$ free-induction decay

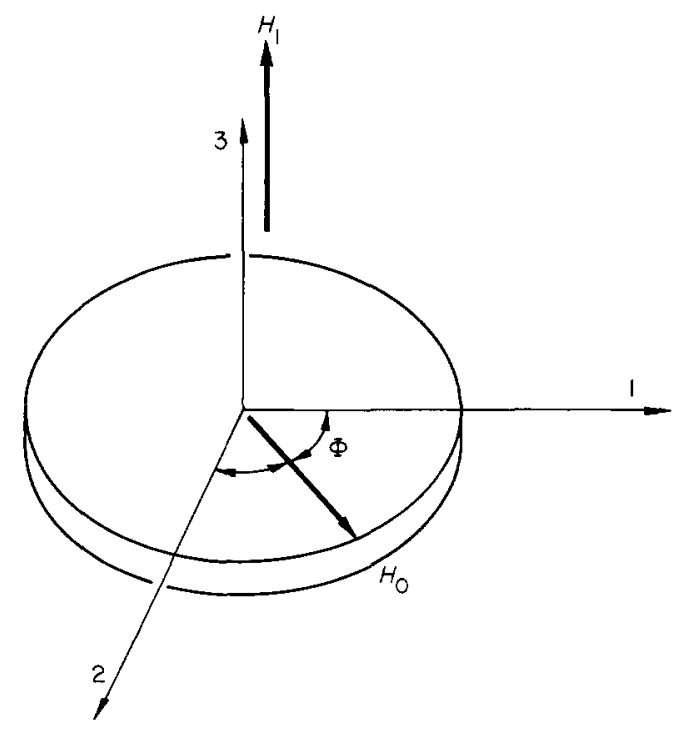

Figure 1 Sketch of the biaxially oriented samples. Orthogonal directions 1,2,3 are defined as follows: 1 is the draw ax is in the plane of the film, and 3 is the normal to the plane of the film. The static magnetic field $H_{0}$ is parallel to the film and makes an angle $\Phi=45$ degrees with the draw axis 1 (in ref. 7 it is shown that for a sample rolled without poling field the orientation of the crystal axes are $c\|1, b\| 2$ and $a \| 3$ )
$(F I D)$ is longer than that for the proton, which decreases the effect of spectrometer dead time.

The polymer sample in the n.m.r. coil was located in a furnace $^{8}$ whose temperature was adjusted with $1^{\circ} \mathrm{C}$ accuracy between room temperature and $145^{\circ} \mathrm{C}$. Sample temperature was measured with a type $K$ thermocouple in contact with the sample holder.

After several attempts using the $\pi / 2-\tau-\pi / 2\left(90^{\circ}\right)$ solid echo sequence, preceded in some cases by a $T_{1 \rho}$ spin locking sequence, it became clear that this procedure would not be effective in removing the amorphous component, especially at temperatures above room temperature where the splitting of the $T_{1 \rho}$ components becomes smaller ${ }^{5}$. Thus, the n.m.r. linewidth was determined from an analysis of the composite $F I D$.

The FID signals stored in a Biomation $2805 \mathrm{M}$ waveform digitizer at $0.2 \mu \mathrm{s} /$ channel were transferred after each sequence to a Northern Scientific NS 575 signal averager. Due to the input impedance of the NS 575 the resultant total dead time was $10 \mu \mathrm{s}$. On averaging over 512 sequences we obtained a signal/noise ratio of about 300.

The study of the longitudinal relaxation time $T_{1}$ was performed using the repetitive saturation recovery sequence $(\pi / 2-\tau-\pi / 2-\tau \ldots)$ with variable delay $\tau$, and $T_{1}$ was determined from the plot of $\log [M(\infty)-M(\tau)]$ vs. $\tau$. In actual fact, a better accuracy in the determination of $T_{1}$ is obtained by integrating the $F I D$ signals $M(t, \tau)$ over a certain time interval $t$. However, because the effective dead time in this experiment was comparable with the $T_{2}$ of the crystalline phase, such a procedure was able to provide reliable information only for the longitudinal relaxation time of the amorphous phase, $T_{1 \mathrm{a}}$. Thus we used for $M_{\mathrm{a}}(\tau)$ an integration over the time interval $30 \mu \mathrm{s}$ $100 \mu \mathrm{s}$ in which the $F I D$ signal from the crystalline phase is almost negligible (a few per cent at room temperature, and a maximum of $15 \%$ at $140^{\circ} \mathrm{C}$ ).

Ih the second sereies of experiments, in Grenoble, we used a home-made pulse n.m.r. spectrometer operating at $20.0 \mathrm{MHz}$ to study the ${ }^{19} \mathrm{~F}$ resonance and especially the spin-lattice relaxation time in the crystalline phase: $T_{1 \mathrm{c}}$ from $10^{\circ} \mathrm{C}$ to $135^{\circ} \mathrm{C}$. A gated integrator was used to average the signals $M(t, \tau)$ from $t=7 \mu$ s to $t=14 \mu$ s after $\pi-$ $\tau-\pi / 2$ pulse sequence. (The dead time was about $6 \mu \mathrm{s}$.) The control of the pulse sequence as well as the accumulation of data (over 20 sequences) was carried out with a homemade computer system based on INTEL SBC cards.

To emphasize the properties of the crystalline phase the highest possible crystallinity of the specimens was desirable and reproducible results were obtained after three successive cycles of measurements (which simultaneously assured annealing) between room temperature and $135^{\circ} \mathrm{C}$.

\section{RESULTS AND DISCUSSION}

\section{Analysis of the free induction decay vs. temperature}

A single method of analysis of the FID observed between room temperature and $145^{\circ} \mathrm{C}$ can be made assuming a simple superposition of two components: a short component attributed to ${ }^{19} \mathrm{~F}$ nuclei in the crystalline phase of the polymer and a longer component attributed to ${ }^{19} \mathrm{~F}$ nuclei in the amorphous (quasi-liquid) phase in which the crystallites are embedded. Such an analysis ${ }^{5}$ appears unambiguous at high temperature $\left(T>80^{\circ} \mathrm{C}\right)$, where the amorphous component clearly 


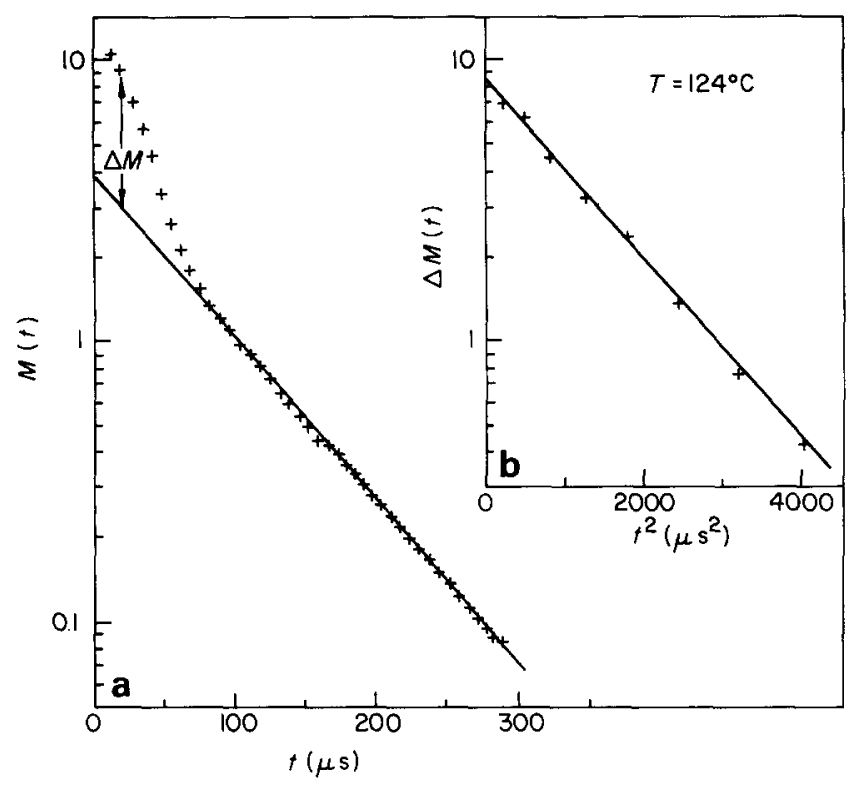

Figure 2 (a) Semi-logarithmic plot of the FID at high temperature, showing decomposition into an exponential $F I D$ attributed to the amorphous phase, and a shorter component $\Delta M(t)$ attributed to the crystalline phase. (b) Analysis of $\log (\Delta M(t))$ vs. $t^{2}$ showing a reasonable agreement with Gaussian behaviour. The crystallinity of the sample at this temperature $\left(124^{\circ} \mathrm{C}\right)$ is evaluated from the ratio $\Delta M(0) / M(0)$

exhibits a Lorentzian line shape typical of viscous liquids (an exponential FID over nearly two decades, see Figure 2) while the crystalline component $\Delta M(t)$ can be described by the Gaussian form

$$
\Delta M(t)=\Delta M(0) \exp \left(-M_{2 \mathrm{c}} t^{2} / 2\right)
$$

where $M_{2 \mathrm{c}}$ is the second moment of the line from the crystal phase.

At lower temperature, and especially around room temperature, the above analysis is ambiguous for two reasons:

(1) The transverse relaxation time of the amorphous phase $T_{2 a}$ strongly decreases as the glass transition temperature is approached $\left(T_{\mathrm{g}}\right.$ is about $\left.0^{\circ} \mathrm{C}^{11}\right)$ and there is no theoretical reason for the corresponding n.m.r. line to retain a Lorentzian shape as $T_{2 a}$ reaches typical values observed in solids.

(2) At room temperature the shorter component of the FID no longer retains a Gaussian form, but exhibits oscilliations which can be described by the phenomenological expression given by Abragam ${ }^{9}$ :

$$
\Delta M(t)=\Delta M(0) \exp \left(-a^{2} t^{2} / 2\right) \sin (b t) / b t
$$

with a second moment $M_{2 \mathrm{c}}=a^{2}+b^{2} / 3$. This behaviour has been observed in the homopolymer $\mathrm{PVF}_{2}$ at room temperature ${ }^{5}$, and is attributed to freezing out of the motions which cause the Gaussian lineshape, leaving only the rigid-lattice dipole interactions which give a lineshape 'in remarkable agreement with the above expression'?

A numerical fitting procedure with the above expression also requires a reliable analytical expression for the amorphous component (or a polynomial expansion ${ }^{10}$ ) and the number of parameters to be fitted is at least 6 . From our data it appeared that trial computer fits give no greater reliability over the whole temperature range than the simple graphical analysis in terms of a Gaussian FID plus an exponential $F I D$, as shown in Figure 2.

In this discussion we also have to stress that whatever the model chosen for describing each phase, such an analysis is based on the assumption of two homogeneous phases, while the morphology of such polymers is known to consist of small lamellar crystals embedded in a partly oriented amorphous phase (see Figure 5). Thus in a more detailed analysis than we present here, one should consider, for the amorphous phase, a distribution of $T_{2 \mathrm{a}}$ values instead of a single (mean) value, which could produce some differences in the evaluation of the crystallinity from each composite FID.

The last difficulty in interpreting our data arises from the change in crystallinity of the sample due to the heat treatment undergone by the polymer during the experiment. This change in crystallinity modifies the relative intensity of the amorphous and crystalline components in the n.m.r. signal and may possibly affect the linewidth and the longitudinal relaxation behaviour. Initially, the quenched sample at room temperature had a crystallinity evaluated at about $40 \%$ from our simple analysis of the n.m.r. results, while it showed a crystallinity of about $50 \%$ from X-ray studies at Thomson Labs. ${ }^{11}$ Given the difference between these techniques and the fact that these two evaluations were not performed on the same specimen, such a discrepancy is not considered significant. Our evaluation of the n.m.r. results in terms of a Gaussian FID plus an exponential FID shows that during the heating necessary to perform the experiment (12 steps of about $10 \mathrm{~K}$ and $2 \mathrm{~h}$ each) the relative intensity of the crystalline signal starts to increase at about the temperature of the rolling process $\left(70^{\circ} \mathrm{C}\right)$, reaching $65 \%$ just before the transition occurs (see Figure $3 a$ ). During heating and cooling in the high temperature phase no significant change in the crystallinity was observed but another increase up to $85 \%$ occurred on cooling through the transition temperature $T_{c}$. These changes in crystallinity and some associated changes in the lamellar morphology were also observed using the small-angle X-ray scattering technique ${ }^{12}$.

In addition, both $T_{\mathrm{c}}$ and the specific heat anomaly at $T_{\mathrm{c}}$ are quite different for the first heating and the second heating ${ }^{3}$ (see also Figure $3 c$ ). However, further experiments on the same samples would be needed for a better understanding of all these results.

Taking into account all these considerations, the evaluation of our n.m.r. results leads to the linewidths plotted in Figure $3 b$ for the amorphous and crystalline components of the signal. The transverse relaxation rate of the nuclei in the amorphous phase $\left(T_{2 \mathrm{a}}\right)^{-1}$ exhibits essentially the same behaviour upon heating and cooling, while the linewidth for the crystalline phase narrows by a factor of approximately 2 around $100^{\circ} \mathrm{C}$ upon heating and returns to its initial value with a hysteresis of $40^{\circ} \mathrm{C}$ upon cooling.

Comparison of this result with the d.s.c. thermograms recorded on the same specimen shows that these changes of linewidth are clearly associated with the anomalies of the specific heat at the ferroelectric transition. From the structural change observed at the transition ${ }^{3}$, it is known that the polymer chains are ordered in a planar all-trans conformation in the low-temperature $\beta$ phase (with a spontaneous polarization perpendicular to the chain axis) and become disordered around the chain axis in irregular $T G T \bar{G}$ and $T T$ sequences in the high-temperature 

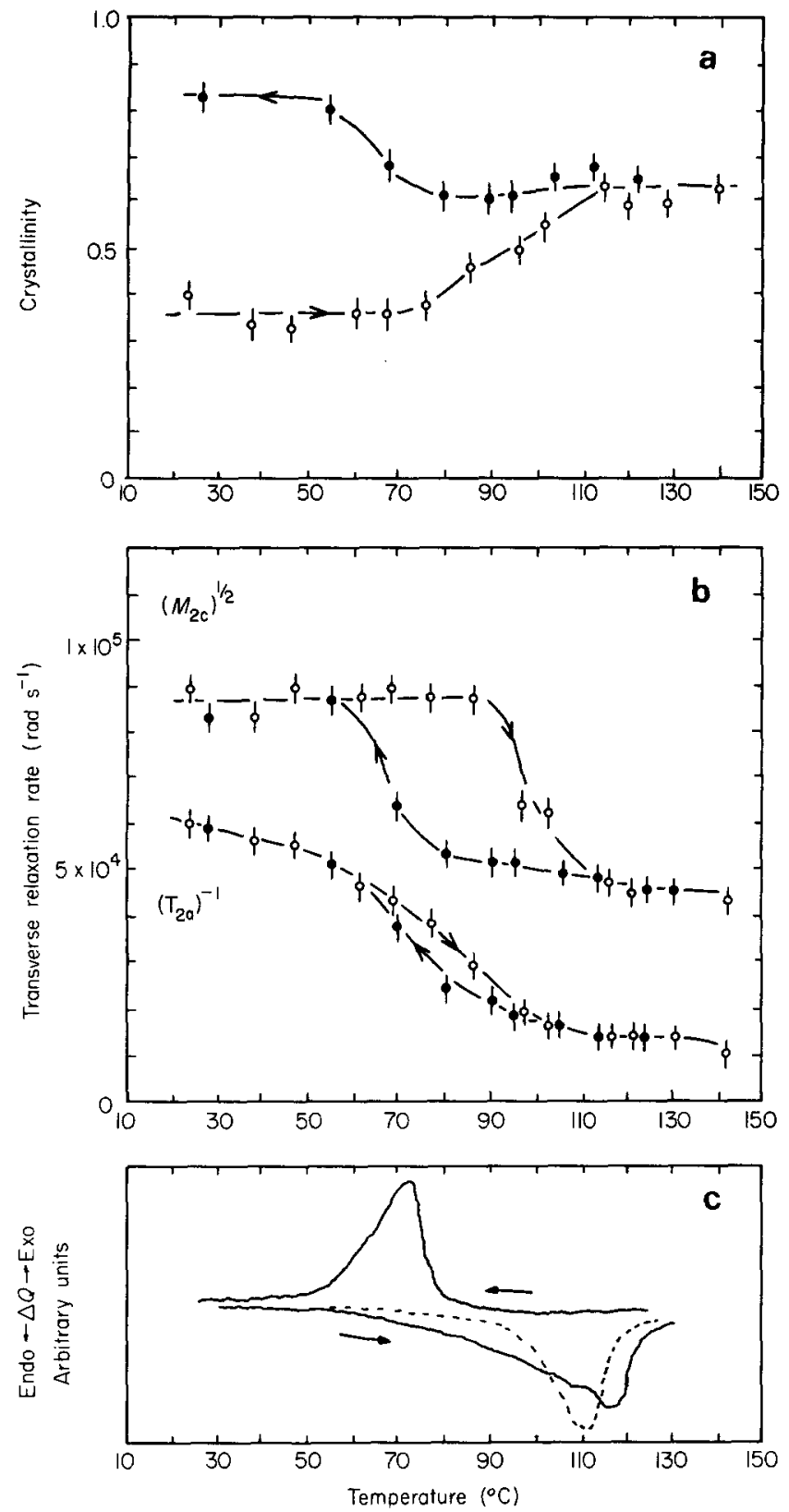

Figure 3 (a) ${ }^{19} \mathrm{~F}$ n.m.r. evaluation of the change in crystallinity for the first heating and cooling cycle. Measurements (at $9.14 \mathrm{MHz}$ ) were made at $2 \mathrm{~h}$ intervals. (b) Temperature dependence of the transverse relaxation rate (as linewidth) defined as $1 / T_{2 \mathrm{a}}$ for the signal of the amorphous phase and as $\left(M_{2 c}\right)^{1 / 2}$ for the signal of the crystalline phase. (c) Differential scanning calorimetry thermograms recorded on the same sample with a Perkin-Elmer Type DCS2C instrument. The full lines are for the first heating and cooling and the dotted line for the second heating (heating and cooling rates of $\pm 5 \mathrm{~K} \mathrm{~min}^{-1}$ )

paraelectric phase $\left(T>T_{\mathrm{c}}\right.$ ). Our present results show that this disorder, which creates a motional narrowing of the n.m.r. line, is of dynamical origin and that the orderdisorder ferroelectric phase transition in this copolymer is comparable to some plastic phase transitions observed in molecular crystals.

Comparable motional narrowing by a factor of about 2 has been observed for the phase changes in benzene ${ }^{13}$, iodoadamantane ${ }^{14}$, tanane $e^{15}$, etc, when the motion taking place in the high-temperature phase is uniaxial, while stronger narrowing has been observed when the motion is endospherical as in $\mathrm{NH}_{4} \mathrm{Cl}^{13}$, adamantane, and fluoroadamantane $\mathrm{e}^{14}$.
Due to the pseudohexagonal symmetry of the low temperature phase the chain motion in the high temperature phase probably consists of $60^{\circ} \mathrm{C}$ (or $120^{\circ} \mathrm{C}$ ) jumps of chain segments around the chain axis, which propagate back and forth like unstable kinks.

Another feature which reinforces the comparison with plastic crystals is the increase of the volume of the unit cell at the order-disorder phase transition ${ }^{1}$.

\section{Spin-lattice relaxation}

The analysis of the spin-lattice relaxation for a material such as this, composed of two types of small intimately connected regions of the same chemical composition (see Figure 5) is simple in two limiting cases: (1) no spin diffusion; (2) fast spin diffusion.

For the case of no spin diffusion, making the approximation that $T_{2 \mathrm{c}}$ and $T_{2 \mathrm{a}}$ each exhibit exponential behaviour, the fractional n.m.r. signal $S\left(t_{0}, \tau\right)$ at a time $t_{0}$ after the end of the second pulse, with a time interval $\tau$ between $\pi / 2$ pulses, is given by

$$
\begin{aligned}
S\left(t_{0}, \tau\right)= & f_{\mathrm{c}} \exp \left(-t_{0} / T_{2 \mathrm{c}}\right)\left[1-\exp \left(-\tau / T_{1 \mathrm{c}}\right)\right] \\
& +f_{\mathrm{a}} \exp \left(-t_{0} / T_{2 \mathrm{a}}\right)\left[1-\exp \left(-\tau / T_{1 \mathrm{a}}\right)\right]
\end{aligned}
$$

where $f_{\mathrm{c}}$ and $f_{\mathrm{a}}$ are the mole fractions of crystalline and amorphous material respectively, and $T_{1 \mathrm{c}}$ and $T_{1 \mathrm{a}}$ are the spin-lattice relaxation times for these materials.

We see that $S\left(t_{0}, \infty\right)-S\left(t_{0}, \tau\right)$ is the sum of two exponentials from which $T_{1 \mathrm{c}}$ and $T_{1 \mathrm{a}}$ can be found. Determination of $f_{\mathrm{c}}$ and $f_{\mathrm{a}}$ requires that $T_{2 \mathrm{c}}$ and $T_{2 \mathrm{a}}$ be known also, because measurements cannot be made for $t_{0}=0$ owing to the receiver dead time.

For the case of fast spin diffusion, the crystalline and amorphous spin populations are completely mixed at all times, and the spin-lattice relaxation recovery function has only one effective relaxation time $T_{1}$ :

$$
\begin{aligned}
S\left(t_{0}, \infty\right) & -S\left(t_{0}, \tau\right) \\
= & {\left[f_{\mathrm{c}} \exp \left(-t / T_{2 \mathrm{c}}\right)+f_{\mathrm{a}} \exp \left(-t_{0} / T_{2 \mathrm{a}}\right)\right] \exp \left(-\tau / T_{1}\right) }
\end{aligned}
$$

where $\left(T_{1}\right)^{-1}=f_{\mathrm{c}} / T_{1 \mathrm{c}}+f_{\mathrm{a}} / T_{1 \mathrm{a}}$

Now from an experimental point of view it is far from obvious to draw conclusions about spin diffusion especially when $T_{1 \mathrm{c}}$ and $T_{1 \mathrm{a}}$ have the same order of magnitude, because in this case both expressions (1) and (2) can be fitted to the same experimental data. Also the size of the crystalline and amorphous regions (typically $100 \AA$ (Figure 5)) is consistent with the possibility of spin diffusion between the two phases.

In spite of these difficulties in interpreting the measurements performed at $9.14 \mathrm{MHz}$ and at $20 \mathrm{MHz}$ we can present two series of data:

With the first apparatus the dead time after the pulse was longer, partly because of the lower frequency. To analyse the saturation recovery at $9.14 \mathrm{MHz}$ we integrated the FID over the time interval 30 to $100 \mu$ s after the pulse. In this interval the amorphous component contributes 80 to $90 \%$ of the FID, so we consider this a measurement essentially of $T_{1 \mathrm{a}}$ for the amorphous phase (' $T_{1 \mathrm{a}}$ ' in Figure 4). This assumption holds in the case without spin diffusion, but in the case of fast spin diffusion it holds only if $T_{1 \mathrm{c}} \gg T_{1 \mathrm{a}}$. The main change in ' $T_{1 \mathrm{a}}$ ' with temperature at $9.14 \mathrm{MHz}$ is a regular increase from $20 \mathrm{~ms}$ 


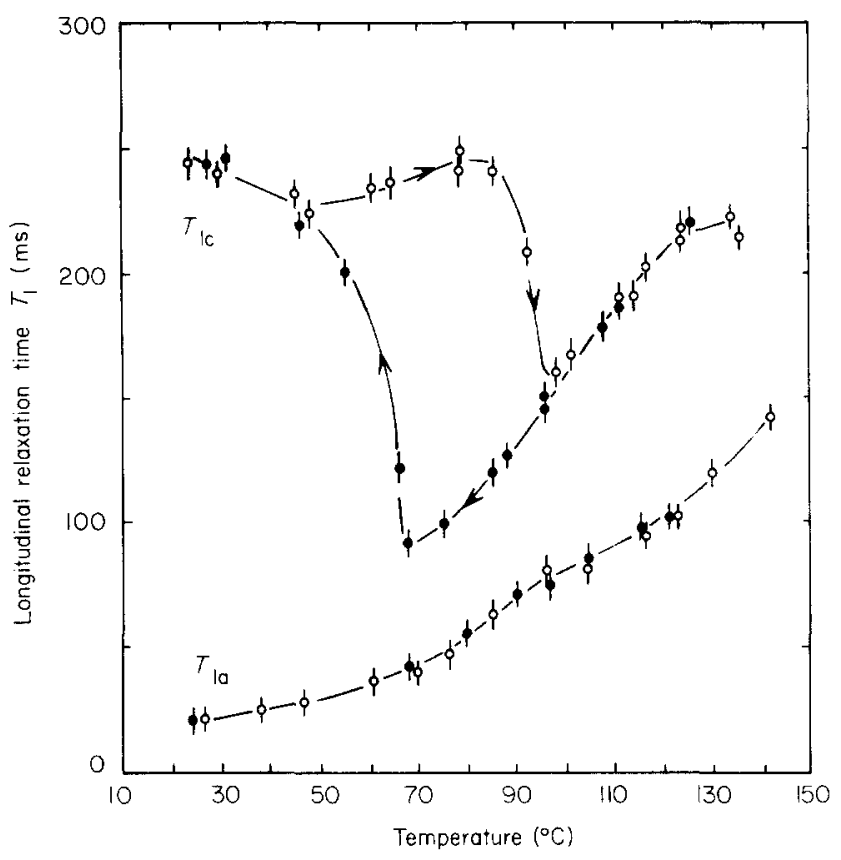

Figure 4 Temperature dependence of the spin lattice relaxation. (a) ' $T_{1 \mathrm{a}}$ ' dominated by the dynamics of the amorphous phase from measurements at $9.14 \mathrm{MHz}$ (first heating and cooling cycle). (b) ' $T_{1 \mathrm{c}}$ ' dominated by the dynamics of the crystalline phase from measurements at $20 \mathrm{MHz}$ (fourth heating and cooling cycle). Circles are for heating and dots for cooling

at $20^{\circ} \mathrm{C}$ to $140 \mathrm{~ms}$ at $140^{\circ} \mathrm{C}$, which can be attributed to a change in the rate of the amorphous phase relaxation mechanism with an activation energy $E_{\mathrm{a}}$ of about $4 \mathrm{kcal} \mathrm{mol}^{-1}$. Also no significant thermal hysteresis was observed while the mole fraction of amorphous material $f_{\mathrm{a}}$ changed from about 0.6 to about 0.15 (see Figure $3 a$ ).

Using the $20 \mathrm{MHz}$ spectrometer, it was possible to
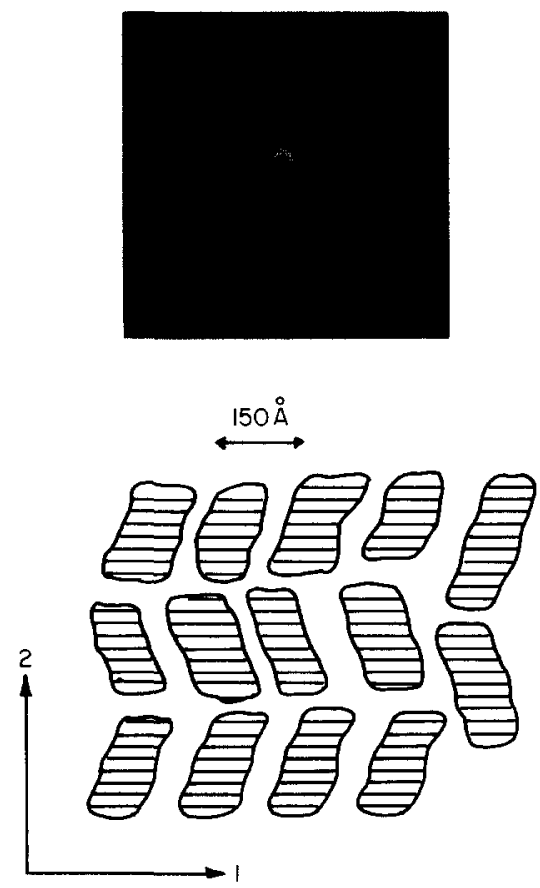

Figure 5 Small-angle $X$-ray scattering pattern from the specimen oriented by rolling and before annealing. (The incident $X$-ray beam is along the normal 3-axis.) Schematic representation of the corresponding lamellar morphology. Inside the crystallites the polymer chains are oriented along the 1 -axis enhance the contribution to the signal from the crystalline phase by two means:

(i) Firstly, the shorter dead time allowed integration of the FID from $7 \mu$ s to $14 \mu$ s after the pulse.

(ii) Secondly, the repeated annealing of the sample up to $135^{\circ} \mathrm{C}$ resulted in an increase of the observed longitudinal relaxation time at room temperature, and we interpret this as being due to an increase of the crystallinity up to about $92 \%$ during the first two thermal cycles.

Thus we consider the measurements performed during the third and fourth thermal cycles somewhat representative of the behavour of $T_{1 \mathrm{c}}$ for the crystalline phase (' $T_{1 \mathrm{c}}$ ' in Figure 4). Indeed, while ' $T_{1 \mathrm{a}}$ ' presents no significant anomaly through the ferroelectric transition, ' $T_{1 \mathrm{c}}$ ' presents well defined minima at $100^{\circ} \mathrm{C}$ on heating and $65^{\circ} \mathrm{C}$ on cooling, thus exhibiting the thermal hysteresis of this phase transition. From the analysis of Figure 4 it also appears that while ' $T_{1 \mathrm{c}}$ ' and ' $T_{1 \mathrm{a}}$ ' differ greatly below $T_{\mathrm{c}}$, ' $T_{1 \mathrm{c}}$ ' is approximately twice ' $T_{1 \mathrm{a}}$ ' above $T_{\mathrm{c}}$ and thus follows a comparable activation behaviour with about the same activation energy, $E_{\mathrm{a}} \approx 4 \mathrm{kcal} \mathrm{mol}^{-1}$. From the data obtained at $20 \mathrm{MHz}$ some additional remarks have to be made.

Above the ferroelectric transition temperature, $T_{c}$, the signal of magnetization recovery versus internal time $\tau$ clearly exhibits single exponential behaviour with reproducible values of ' $T_{1 \mathrm{c}}$ ' whatever the thermal cycle considered, i.e. both for the recrystallized sample and for the virgin sample. Two different interpretations of this result can be given, depending on the status attributed to spin diffusion above $T_{\mathrm{c}}$.

If there is fast spin diffusion, expression (2) implies that the crystallinity of the polymer in the high-temperature phase should not depend on the thermal history of the sample. Thus taking $f_{\mathrm{c}}=0.65$ from Figure $3 a$ and assuming for $T_{1 \mathrm{a}}$ the values of ' $T_{1 \mathrm{a}}$ ' measured at $9.14 \mathrm{MHz}$ one gets values of $T_{1 \mathrm{c}}$ about twice those of ' $T_{1 \mathrm{c}}$ ' over the whole paraelectric phase (supposing that $T_{1 \mathrm{a}}$ does not depend on frequency).

If there is no spin diffusion, reasonable fits with expression (1) can also be obtained. For a crystallinity larger than $f_{\mathrm{c}}=0.9$ the resultant values of $T_{1 \mathrm{c}}$ are those plotted in Figure 4 (' $T_{1 \mathrm{c}}$ ') while for a minimum crystallinity of $f_{\mathrm{c}}=0.65$ the values of $T_{1 \mathrm{c}}$ appear at most $20 \%$ larger than ' $T_{1 \mathrm{c}}$ ', taking again for $T_{1 \mathrm{a}}$ the values measured at $9.14 \mathrm{MHz}$.

Below the ferroelectric transition temperature $T_{\mathrm{c}}$ the signal of magnetization recovery versus internal time $\tau$ exhibits a different behaviour which does not fit a single exponential decay indicating that the possibility of fast spin diffusion below $T_{\mathrm{c}}$ can at least be eliminated.

Alternatively, expression (1) can be used for fitting the data of the low temperature phase. Assuming a crystallinity of $f_{\mathrm{c}}=0.92$ we get from the data of the third and fourth thermal cycles the values ' $T_{1 \mathrm{c}}$ ' plotted in Figure 4. However, these fits also give values of $T_{1 \mathrm{a}}$ about twice those measured at $9.14 \mathrm{MHz}$ (' $T_{1 \mathrm{a}}$ '), not only for the recrystallized sample but also for the virgin sample at room temperature (assumed crystallinity $f_{c}=0.5$ ).

It thus appears that the spin lattice relaxation time in the amorphous phase $T_{1 \mathrm{a}}$ approximately doubles on doubling the frequency of the measurement. Such a result, if it holds above $T_{\mathrm{c}}$, implies that at $20 \mathrm{MHz} T_{1 \mathrm{a}} \approx$ ' $T_{1 \mathrm{c}}$ ' and this could explain the apparent single exponential behaviour observed in the high-temperature phase without 
the need for invoking fast spin diffusion or a constant value of the crystallinity.

From the above discussion we can conclude that:

(1) In the ferroelectric phase $\left(T<T_{\mathrm{c}}\right)$ only weak spin diffusion is consistant with our results at $9.14 \mathrm{MHz}$ and $20 \mathrm{MHz}$, which show a large difference between spinlattice relaxation in the two phases of the polymer.

(2) In the high-temperature crystalline phase the spinlattice relaxation is closer to that in the amorphous phase but the role of spin diffusion in mixing the thermal reservoirs of these two disordered phases is not yet clear from the present results. Indeed the change in the crystallinity and in the lamellar morphology ${ }^{12}$ observed at the transition may change the relative effect of the spin diffusion at $T_{c}$.

\section{CONCLUSIONS}

We have demonstrated in this preliminary study that the ferroelectric phase transition in the crystalline phase of copolymers of vinylidene fluoride and trifluoroethylene is accompanied by a motional narrowing of the ${ }^{19} \mathrm{~F}$ n.m.r. line and a definite anomaly in $T_{1 \mathrm{c}}$. It thus follows that the high-temperature phase is dynamically disordered. In the high-temperature phase each segment of the molecule has several possible equilibrium positions, which results in a higher statistical symmetry of the crystalline structure (point group 6/mmm or $\overline{6} 2 \mathrm{~m}$ ?). Freezing of this motion at $T_{\mathrm{c}}$ leads to an ordered structure with cooperative alignment of the dipole moments along one of the six (or three) possible axes, which may result in six (or three) possible ferroelectric domains in the crystallites when the transition is obtained by cooling without applied electric field.

Both the $T_{1 \mathrm{c}}$ and $T_{2 \mathrm{c}}$ measurements clearly show the relative sharpness of the ferroelectric transition and its large thermal hysteresis. These measurements also show that n.m.r. can be used to estimate the degree of crystallinity in this polymer and follow its increase during the annealing process.

It appears that the n.m.r. technique, which has proved to be very fruitful for studying the phase transitions in materials like ammonium chloride or hydrogen-bonded ferroelectrics, presents several difficulties when one at- tempts to adapt it to such a semicrystalline material with at least two phases. In regard to this, a theoretical study of the effect on $T_{1 \mathrm{a}}$ and $T_{1 \mathrm{c}}$ of spin diffusion between nuclei in the amorphous and crystalline phases is recommended. Further studies of crystallinity using $T_{2}$ measurements and of the molecular motion using $T_{1 \mathrm{c}}$ measurements at higher frequencies would help clarify phenomena associated with the ferroelectric phase transition in these copolymers.

\section{ACKNOWLEDGEMENTS}

We thank François Micheron of Thomson-CSF for providing the copolymer samples. Robert Blinc kindly assisted with some of the n.m.r. measurements. One of us (JFL) acknowledges support from a NATO Fellowship. This work was supported in part by National Science Foundation Grant No. DMR-8 205280.

\section{REFERENCES}

1 Davis, G. T., Furukawa, T., Lovinger, A. J. and Broadhurst, M. G. Macromolecules 1982, 15, 323, 329

2 Lovinger, A. J., Furukawa, T., Davis, G. T. and Broadhurst, M. G. Ferroelectrics $1983,50,553$

3 Lovinger, A. J., Furukawa, T., Davis, G. T. and Broadhurst, M. G. Polymer 1983, 24, 1225, 1233

4 Legrand, J. F. and Lajzerowicz, J. Ferroelectrics 1983, 51, 129

5 McBrierty, V. J., Douglass, D. C. and Weber, T. H. J. Polym. Sci. Polym. Phys. Edn. 1976, 14, 1271

6 Douglass, D. C., McBrierty, V. J. and Wang, T. T. J. Chem. Phys. $1982,77,5826$

7 Servet, B., Ries, S., Broussoux, D. and Micheron, F. J. Appl. Phys. $1984,55,2763$

8 Schmidt, V. H. and Schuele, P. J. Bull. Am. Phys. Soc. 1982, 27, 189

9 Abragam, A. 'The Principles of Nuclear Magnetism', Oxford University Press, London, 1961, p. 120

Meurer, B., Spegt, P. and Weill, G. J. Phys. E 1983, 16, 403

Broussoux, D. and Micheron, F. (private communication)

Delzenne, P. and Legrand, J. F. (private communication)

Ref. 9, pp. 451-2

Amoureux, J. P. Thèse d'Etat (1980), (unpublished)

Bordeaux, D., Capiomont, A., Lajzerowicz, J., Jouve, M. and Thomas, M. Acta Crystallogr. B 1974, 30, 2156

16 Ishii, F., Sawatari, T. and Odajima, A. Jpn. J. Appl. Phys. 1982, 21, L251

17 McBrierty, V. J., Douglass, D. C. and Furukawa, T. Macromolecules 1984, 17, 1136 